\title{
Application of digital mammography-based radiomics in the differentiation of benign and malignant round-like breast tumors and the prediction of molecular subtypes
}

\author{
Lanyun Wang $^{1 \#}$, Wenjun Yang ${ }^{1 \#}$, Xiaoli Xie ${ }^{2}$, Weiyan Liu ${ }^{3}$, Hao Wang ${ }^{1}$, Jinjiang Shen ${ }^{1}$, Yi Ding ${ }^{1}$, \\ Bei Zhang ${ }^{4}$, Bin Song'
}

${ }^{1}$ Department of Radiology, Minhang Branch, Zhongshan Hospital, Fudan University, Shanghai, China; ${ }^{2}$ Department of Pathology, Minhang Branch, Zhongshan Hospital, Fudan University, Shanghai, China; ${ }^{3}$ Department of General Surgery, Minhang Branch, Zhongshan Hospital, Fudan University, Shanghai, China; ${ }^{4}$ Department of Radiology, Ruijin Hospital, Shanghai Jiao Tong University School of Medicine, Shanghai, China

Contributions: (I) Conception and design: L Wang, B Zhang, B Song; (II) Administrative support: B Song; (III) Provision of study materials or patients: All authors; (IV) Collection and assembly of data: All authors; (V) Data analysis and interpretation: L Wang; (VI) Manuscript writing: All authors; (VII) Final approval of manuscript: All authors.

\#These authors contributed equally to this work.

Correspondence to: Bin Song. Department of Radiology, Minhang Branch, Zhongshan Hospital, Fudan University, Shanghai 201199, China. Email: sb72778@189.cn; Bei Zhang. Department of Radiology, Ruijin Hospital, Shanghai Jiao Tong University School of Medicine, Shanghai 200025, China. Email: zhangbei650213@126.com.

Background: This study aimed to investigate the diagnostic performance of radiomic features based on digital mammography (DM) in the differential diagnosis of benign and malignant round-like (round and oval) solid tumors with circumscribed or obscured margins but without suspicious malignant or benign macrocalcifications and to investigate whether quantitative radiomic features can distinguish triple-negative breast cancer (TNBC) from non-TNBC (NTNBC).

Methods: This retrospective study included 112 patients with round-like tumors who underwent DM within 20 days preoperatively. Breast masses were segmented manually on the DM images, then radiomic features were extracted. The predictive models were used to distinguish between benign and malignant tumors and to predict TNBC in invasive ductal carcinoma. The receiver operating characteristic curves (ROCs) for these models were obtained for initial DM characteristics, radiomic features to predict malignant tumors and TNBC. The decision curve was obtained to evaluate the clinical usefulness of the model for the prediction of benign or malignant tumors.

Results: The study cohort included 79 patients with pathologically confirmed malignant masses and 33 patients with benign (training cohort: $n=79$; testing cohort: $n=33$ ). A total of 396 features were extracted from the DM images for each patient. The radiomics model for the prediction of malignant tumors achieved an area under the receiver operating characteristic curve (AUC) of 0.88 [95\% confidence interval (CI), 0.761.00] in the testing cohort; the radiomics model for the prediction of TNBC achieved an AUC of 0.84 (95\% CI, 0.73-0.96). In contrast, DM characteristics alone poorly predicted malignant tumors, with the density achieving an AUC 0.69 (95\% CI, 0.59-0.79); there was no significant difference in DM characteristics between TNBC and NTNBC $(\mathrm{P}>0.05$, all). The decision curve showed the good clinical usefulness of the model for the prediction of malignant tumors.

Conclusions: This study showed that DM-based radiomics can accurately discriminate between benign and malignant round-like tumors with circumscribed or obscured margins but without suspicious malignant or benign macrocalcifications. Additionally, it can be used to predict TNBC in invasive ductal carcinoma. DM-based radiomics can aid radiologists in mammogram reading, clinical diagnosis and decision-making.

Keywords: Digital mammography (DM); radiomics; round-like breast tumors; molecular subtypes 
Submitted Apr 27, 2020. Accepted for publication Sep 18, 2020.

doi: $10.21037 /$ gs-20-473

View this article at: http://dx.doi.org/10.21037/gs-20-473

\section{Introduction}

Breast cancer is the most common malignant disease diagnosed in women worldwide (1-3). The mortality of breast cancer has decreased significantly since the 1970s (4). The decrease is attributed to both the availability of screening methods, especially mammography, and improved therapy for more advanced cancer. Digital mammography $(\mathrm{DM})$ is a proposed method for detecting breast tumors in clinical practice (5). Breast-Imaging Reporting and Data System (BI-RADS) $(6,7)$ is used worldwide to describe the findings of the various breast-imaging techniques including DM. Although DM plays an important role in the detection of breast masses, there is still overlap in DM image features between benign and malignant breast tumors. Round and oval masses with circumscribed or obscured margins, without suspicious malignant and benign macrocalcifications are difficult to diagnose. The types of tumors include invasive ductal carcinoma (some are TNBCs), intraductal papillary lesions, mucinous adenocarcinomas, phyllodes tumors, intracystic papillary carcinoma, fibroadenomas, etc. $(8,9)$. However, the clinical interventions and prognosis of these types of tumors are different (8).

TNBC is a distinctive subtype of breast cancer that does not express estrogen receptors (ERs), progesterone receptors (PRs) or human epidermal growth factor receptor 2 (HER2) (10). This phenotype of breast cancer displays poor prognosis due to aggressive tumor biology (11), and tends to occur in younger women (12). TNBCs commonly present as benign characteristics on mammogram and ultrasound (US) images and lack the typical representative suspicious mammographic characteristics of breast cancer $(12-14)$. TNBC tend to be misdiagnosed or delayed diagnosis when lack of large positive axillary nodes (15). Therefore, traditional DM may not be the ideal means for identifying TNBC.

According to the previous study (7), breast masses should be examined with US or magnetic resonance imaging (MRI) to decide their ultimate BI-RADS category, except for fatty or completely calcified masses. Breast dynamic contrast-enhanced MRI has high accuracy in identifying benign and malignant breast tumors (16), but it requires contrast medium injection and is more expensive. US is an indispensable tool in breast imaging (17). To some extent, US examination for breast cancer diagnosis is neither adequately sensitive nor sufficiently specific (18) and is operator dependent.

Radiomics can convert medical images into highdimensional, mineable data via high-throughput extraction of quantitative features and allows for quantitative evaluation of tumor heterogeneity (19). DM-based radiomics has been studied for predicting the classification of microcalcification (20) and molecular subtypes of breast cancer $(21,22)$. Applications of DM image texture analysis in the differential diagnosis of benign and malignant breast tumors have also been reported (23). DM is the most common examination for breast cancer screening and diagnosis, while no quantitative parameter may be gained by the analysis of DM images (23). Therefore, finding a quantitative biomarker from DM is greatly needed to increase the diagnostic power in breast tumors. In this study, we aimed to evaluate the diagnostic performance of DM-based radiomics in the differential diagnosis between benign and malignant round-like tumors. Furthermore, we investigated whether DM-based radiomics can well distinguish TNBC from NTNBC. DM-based radiomics may be helpful for radiologists in mammogram reading and clinical diagnosis of round-like tumors. We present the following article in accordance with the STARD reporting checklist (available at http://dx. doi. org/10. 21037/gs-20473).

\section{Methods}

\section{Patients}

The study was conducted in accordance with the Declaration of Helsinki (as revised in 2013). This retrospective study was approved by the institutional review board of Minhang Branch, Zhongshan Hospital (approval number: 2020-012-01K), and informed consent was waived. All patients who underwent DM examination with pathologically confirmed benign (excluding fibroadenoma) and malignant tumor were continuously retrospectively collected from January 2017 to December 2019. The patients who underwent DM examination with 
pathologically confirmed fibroadenoma were continuously retrospectively collected from June to December 2019 because of the large number of patients with fibroadenoma. Data from above all patients were retrieved from the Picture Archiving and Communication System (PACS) at the Affiliated Minhang Hospital of Fudan University. The clinical characteristics included age, sex and pathologic results. The inclusion criteria were as follows: (I) the shape of the tumor was oval or round; (II) DM examination was performed within 20 days preoperatively, and imaging quality met the requirements for postprocessing; (III) benign or malignant breast tumors were confirmed by histopathologic examination. The exclusion criteria were as follows: (I) before DM examination, patients underwent any treatment including chemotherapy, surgery, radiotherapy, and anti-HER2 therapy; (II) tumors that are not fully displayed in the cranial caudal (CC) or/and mediolateral oblique (MLO) views; (III) tumors with architectural distortion (excluding scarring associated with a previous injury or surgery); (IV) tumors with calcifications of BIRADS category $2 / 4 b / 4 c / 5$; (V) tumors with spiculated margins; (VI) tumors that could not be displayed because the breasts were extremely dense. Finally, 112 patients were included in this study. Figure 1 shows the selection of the study group.

\section{Digital mammography and tumor segmentation}

All data were acquired on a GE Senographe Essential DM system (GE Healthcare, Milwaukee, WI). For each patient, images of one optimal MLO and one optimal CC view were exported as Digital Imaging and Communications in Medicine (DICOM) data. No further processing or normalization was performed on the images.

ITK-SNAP software (http://www.itk-snap.org) was used for the segmentation of breast tumors. The regions of interest (ROIs) were manually segmented on both MLO and CC views by two radiologists (WJY, LYW) with 10 and 14 years of experience in DM imaging, respectively. If the margins of some lesions were obscured, the two radiologists will reach consensuses by reading the images additionally to determine the ROIs.

\section{DM characteristics}

All DM images were reviewed by two radiologists with 14 and 10 years of experience in interpretation of DM images. Decisions on DM images findings were made by consensus.
They recorded the following: (I) margin (circumscribed, obscured); (II) density (low-density, equal-density, highdensity); (III) location (depth) (anterior, middle, posterior). All these DM Characteristics were selected for comparison based on past study guidelines (6).

DM characteristics presented as categorical variables were analyzed by Chi-square test or Fisher exact test. Univariate analysis was applied to select DM risk factors for malignant tumors and TNBC $(\mathrm{P}<0.05)$ respectively. Then, backward stepwise multivariate logistic regression selection and the likelihood ratio test were performed, and the MG models for the prediction of malignant tumors and TNBC were constructed, respectively. The performance of the models was assessed by ROC curve, respectively.

\section{Radiomics for the prediction of benign and malignant tumors}

\section{Feature extraction, selection and model construction}

Radiomic features were automatically extracted using AK software version 3.2.2 (GE healthcare). A total of 396 features were extracted, including histogram, shape, graylevel cooccurrence matrix (GLCM), gray-level run-length matrix (GLRLM), and gray-level size zone matrix (GLSZM) features. To evaluate the interobserver agreement of the extracted features, we randomly selected CC views from 30 patients and calculated the intraclass correlation coefficient (ICC) of the features. According to the $95 \%$ confidence interval of the ICC estimate (24), values greater than 0.90 , between 0.75 and 0.9 , between 0.5 and 0.75 , and less than 0.5 were classified as excellent, good, moderate and poor reliability, respectively. Each feature was utilized for further analysis merely if the ICC value reached 0.75 .

Participants were randomly separated into a training cohort $(n=79)$ and testing cohort $(n=33)$ at a ratio of 7:3. First, we applied the mRMR (maximum correlation minimum redundancy) algorithm to eliminate redundant and irrelevant features based on the training cohort and retained 15 features without redundancy and with high correlation with labels. Then, the least absolute shrinkage and selection operator (LASSO) with ten-fold cross validation was applied to further select the feature subsets through regularization by optimizing the hyperparameter $\lambda$. Coefficients of some candidate features were compressed to zero at the optimum $\lambda$, and features with nonzero coefficients were retained. A radiomics signature was constructed through a linear combination of the nonzero coefficient features. The radiomics score (rad-score) of each 


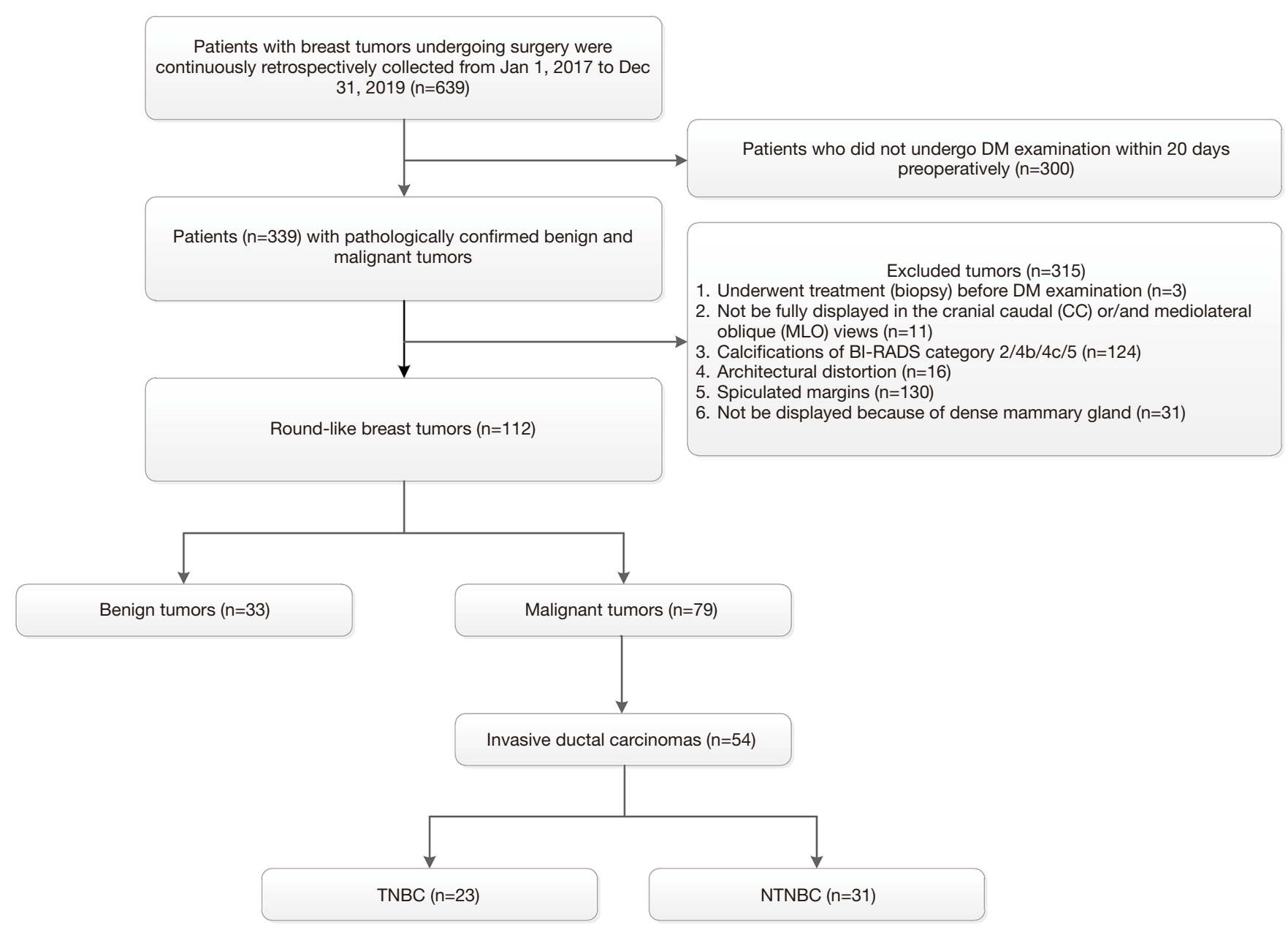

Figure 1 Flow chart of study group selection. DM, digital mammography; CC cranial caudal; MLO, mediolateral oblique; TNBC, triplenegative breast cancer; NTNBC, non-triple-negative breast cancer.

patient was calculated.

\section{Model validation}

The performance of the model for differentiating benign from malignant masses was assessed by receiver operating characteristic (ROC) curve analysis in the training cohort and validated in the testing cohort. The area under the curve (AUC), accuracy, sensitivity, specificity, positive predictive value and negative predictive value were calculated. The radiomic framework is shown in Figure 2.

In addition, we performed 100-folds leave-group-out cross-validation (LGOCV) to verify the reliability of our model and illustrate that the results given in our model are not contingent.

\section{Radiomics for the prediction of TNBC and NTNBC}

Furthermore, we investigated invasive ductal carcinoma, including TNBC and NTNBC, in the malignant patient group. First, for 396 radiomic features, independent t-test or Wilcoxon test was used to compare the difference between TNBC and NTNBC. Second, univariate logistic analysis was applied to assess whether the features were independent predictive risk factors $(\mathrm{P}<0.05)$. Third, mRMR was applied to select the relevant and nonredundant features, and 10 features were retained. Then, backward stepwise multivariate logistic regression selection and the likelihood ratio test were performed, and the final prediction model was constructed. The performance of the model was assessed by ROC curve. Cross-validation was also performed to verify the reliability of our results. 


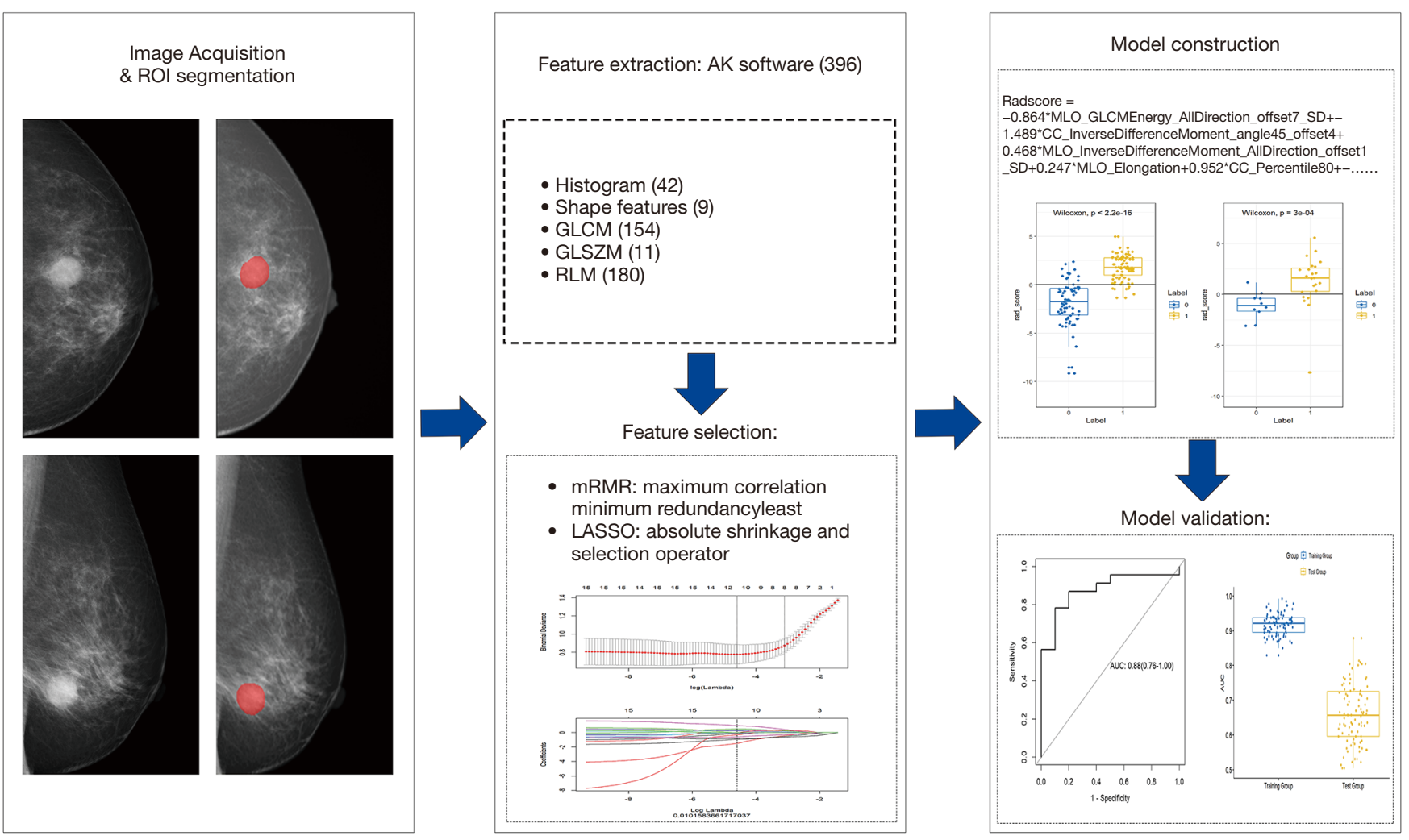

Figure 2 Flow chart of radiomics analysis of breast round-like masses on mammography.

\section{Statistical analysis}

SPSS (v. 23) and R statistical software v. 3.5.1 (https://www. Rproject.org) were performed in the statistical analysis.

\section{Results}

\section{Patient characteristics}

A total of 112 patients (age: $55.87 \pm 14.25$ years; age range: 23-86 years) were included in our study. Among 112 patients, 79 (age: 59.80 13.82 years; age range: $32-86$ years) were included in the malignant group, and 33 (age: $46.50 \pm 10.32$ years; age range: $23-65$ years) were included in the benign group. Table 1 shows the histopathologic types of breast masses enrolled in the study. Fifty-four patients in the malignant group with invasive ductal carcinoma were further divided into two groups, with 23 cases of TNBC and 31 cases of NTNBC.

\section{Benign and malignant tumor prediction}

DM characteristics of the malignant and benign tumors are listed in Table 2. There was significant difference in density between the malignant and benign tumors (Table 2). The lesion density for the prediction of benign or malignant tumors achieved an area under the receiver operating characteristic curve (AUC) of 0.69 [95\% confidence interval (CI), 0.59-0.79]. For predicting benign and malignant masses, 10 top-performing features, 3 from the CC view and 7 from the MLO view, were finally retained to construct the radiomics signature (Table 3). The proportion of features derived from the MLO view was higher (7/10). Table 3 shows the coefficient and $\mathrm{P}$ value of the selected features. All 10 features were significantly different between benign and malignant masses $(\mathrm{P}<0.05)$. Figure 3 shows the difference in radiomics score between benign and malignant masses. The radiomics prediction model achieved an AUC value of $0.94(0.90-0.97)$ in the training set (Figure $4 A)$ and $0.88(0.76-1.0)$ in the testing set (Figure $4 B)$, significantly higher than the AUC value [0.69 (0.59-0.79)] of the lesion density (initial DM characteristic). Figure $4 C$ shows the results of 100 folds LGOCV. Figure 4D shows the clinical decision curve of the radiomics model. Table 4 shows the performance of the rad-score in discriminating between 
Table 1 Histopathologic type of breast masses and description of calcifications

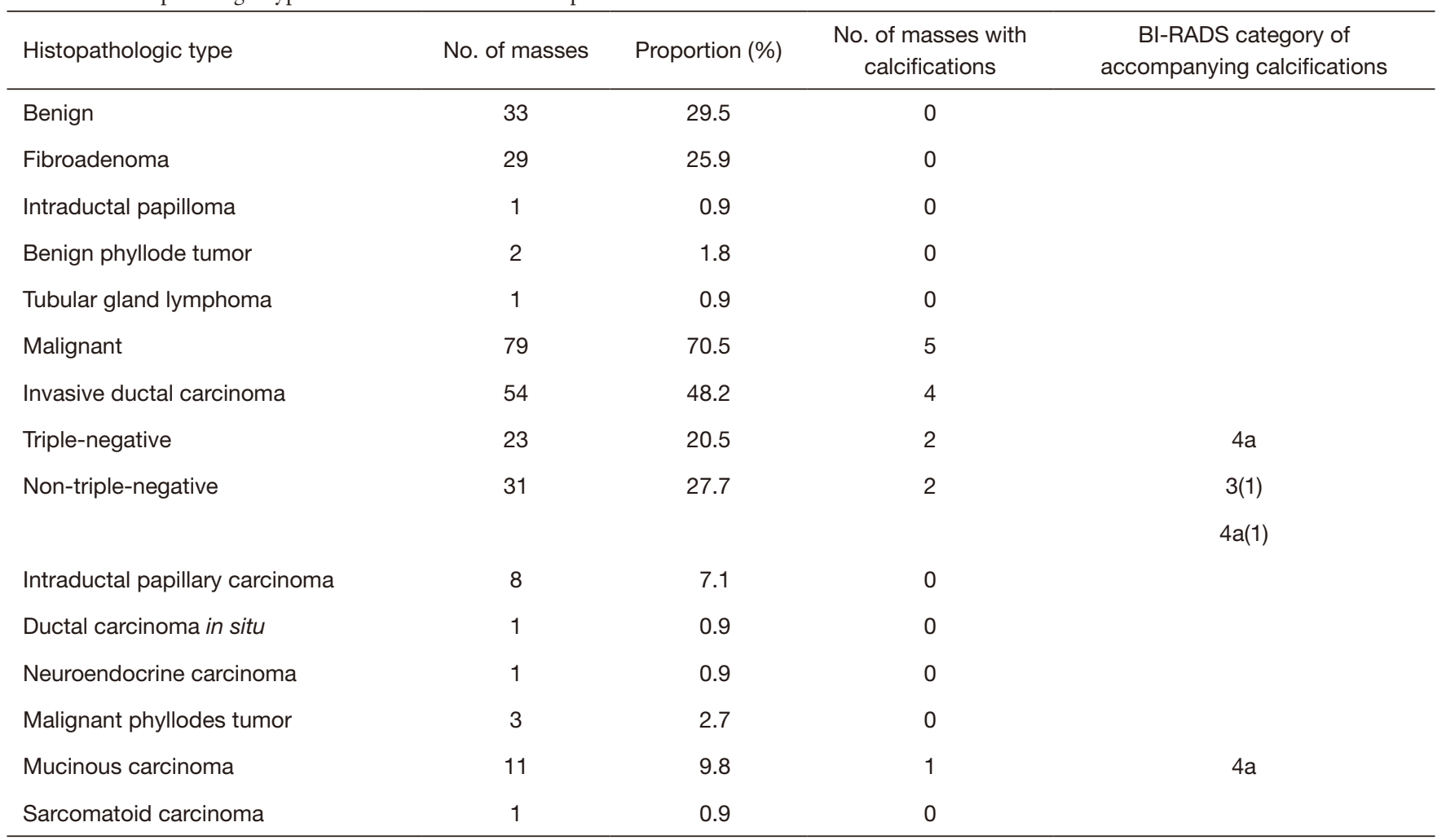

BI-RADS, Breast Imaging Reporting and Data System.

Table 2 DM characteristics for the differential diagnosis of benign and malignant tumors

\begin{tabular}{|c|c|c|c|c|}
\hline Characteristics variables & Benign tumors & Malignant tumors & $\chi^{2}$ value & $P$ value \\
\hline Circumscribed & 12 & 24 & & \\
\hline Obscured & 21 & 55 & & \\
\hline Density & & & 13.297 & $0.001^{*}$ \\
\hline Equal-density & 26 & 34 & & \\
\hline High- density & 6 & 44 & & \\
\hline Location(depth) & & & 5.784 & 0.055 \\
\hline Anterior & 3 & 16 & & \\
\hline
\end{tabular}

DM, digital mammography; ${ }^{*}$ means $\mathrm{P}<0.05$. 
Table 3 Extracted modeling features predictive of benign and malignant tumors

\begin{tabular}{lcc}
\hline Feature variable & Coefficient & P value \\
\hline MLO_GLCMEnergy_AllDirection_offset7_SD & -0.864 & $<1 \mathrm{e}-04^{*}$ \\
CC_Inverse Difference Moment_angle45_offset4 & -1.489 & $0.001^{*}$ \\
MLO_Inverse Difference Moment_AlIDirection_offset1_SD & $0.001^{*}$ \\
MLO_Elongation & 0.468 & $0.003^{*}$ \\
CC_Percentile80 & $<1 \mathrm{e}-04^{*}$ \\
CC_Inverse Difference Moment_All Direction_offset7_SD & 0.952 & $0.000^{*}$ \\
MLO_GLCMEntropy_AllDirection_offset7_SD & -0.917 & $0.011^{*}$ \\
MLO_Low Intensity Large Area Emphasis & -0.107 & $<1 \mathrm{e}-04^{*}$ \\
MLO_Correlation_angle135_offset7 & -0.59 & $<1 \mathrm{e}-04^{*}$ \\
MLO_ClusterShade_angle0_offset7 & -0.105 & $0.043^{*}$ \\
\hline${ }^{*}$ means P<0.05. & -0.312 & \\
\hline
\end{tabular}

${ }^{*}$ means $\mathrm{P}<0.05$.
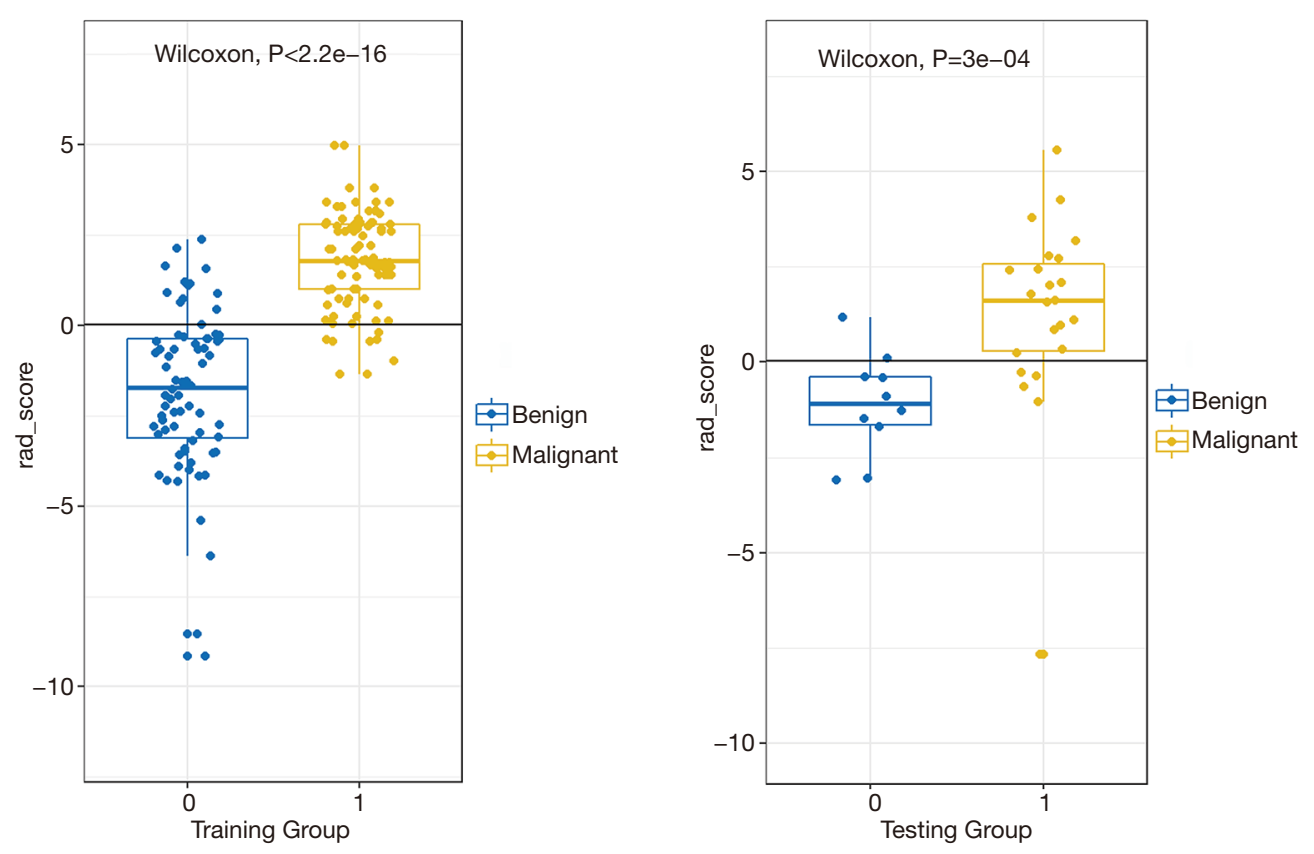

Figure 3 Distribution of radiomics score value of benign and malignant masses in the training and testing groups.

benign and malignant masses, and the positive predictive value achieved $90 \%$ in the test group. These results indicated an overall good performance of the prediction model.

\section{TNBC and NTNBC prediction}

DM characteristics of TNBC and NTNBC are listed in
Table 5. There was no significant difference in margin, density and location between TNBC and NTNBC $(\mathrm{P}>0.05$, all) (Table 5).

For predicting TNBC and NTNBC, 3 features, 1 from the MLO view and 2 from the CC view, were finally selected to construct the prediction model (Table 6). All features were significantly different between TNBC and NTNBC $(\mathrm{P}<0.05)$. Figure 5 show the performance of the 


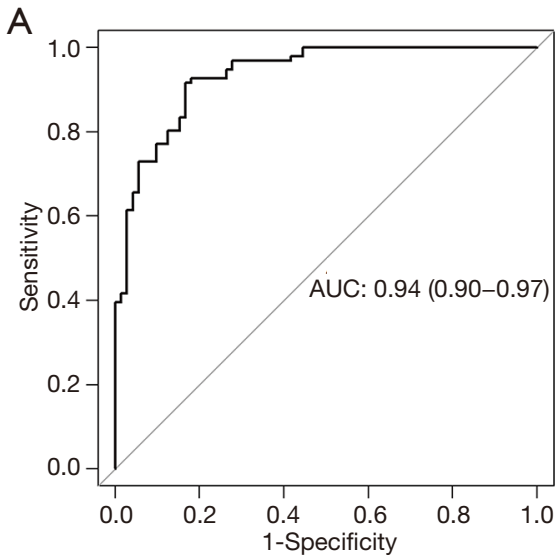

C

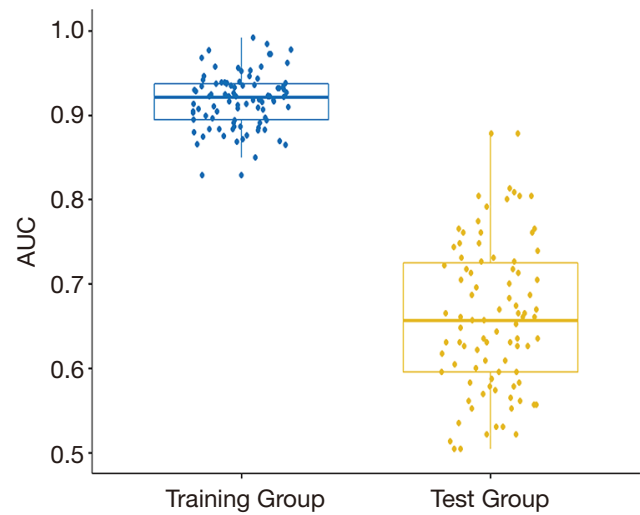

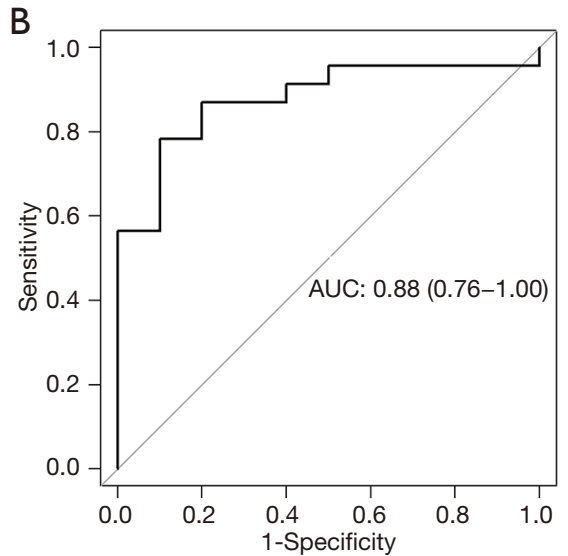

D

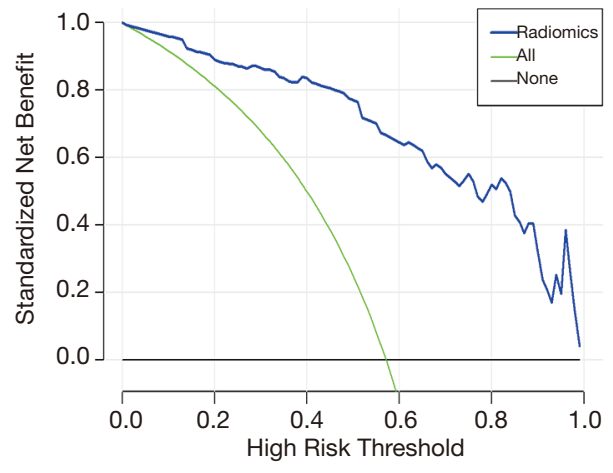

Figure 4 Receiver operating characteristic curves of the radiomics model for predicting benign and malignant masses in the training (A) and testing (B) sets. Boxplot shows the results of 100 folds LGOCV (C). The decision curve of the radiomics model (D) shows that in the threshold range from $0-1$, the radiomics model gains a greater benefit than other cases. LGOCV, leave group out cross-validation.

Table 4 The performance of the radiomic score in differentiating benign from malignant tumors

\begin{tabular}{lccccc}
\hline Group & Accuracy $(95 \% \mathrm{Cl})$ & Sensitivity $(95 \% \mathrm{Cl})$ & Specificity $(95 \% \mathrm{Cl})$ & $\begin{array}{c}\text { Positive predictive value } \\
(95 \% \mathrm{Cl})\end{array}$ & $\begin{array}{c}\text { Negative predictive value } \\
(95 \% \mathrm{Cl})\end{array}$ \\
\hline Training & $0.881(0.822,0.926)$ & $0.912(0.838,0.961)$ & $0.833(0.723,0.907)$ & $0.880(0.796,0.934)$ & $0.882(0.776,0.944)$ \\
Test & $0.788(0.611,0.910)$ & $0.783(0.558,0.917)$ & $0.800(0.442,0.965)$ & $0.900(0.669,0.982)$ & $0.615(0.323,0.849)$ \\
\hline
\end{tabular}

$\mathrm{Cl}$, confidence interval.

prediction model. The AUC value, accuracy, sensitivity and specificity were $0.84(0.73-0.86), 0.81$ (0.68-0.91), 0.78 (0.56-0.93), 0.83 (0.65-0.94), respectively. Table 7 showed the result of the 100 times cross-validation.

\section{Discussion}

Our study showed that DM-based radiomics has better diagnostic ability for round-like breast tumors than original DM characteristics. DM-based radiomics could be used as a noninvasive method for the identification of roundlike benign and malignant tumors and the prediction of molecular subtypes.

DM has precedence for detecting calcifications in breast tumors. However, due to the covering of the glands, the edge of some lesions may be insufficiently displayed in 
DM images. Furthermore, the density of lesions cannot be quantified, and the blood flow cannot be displayed. Therefore, the differential diagnosis of benign or malignant lesions may be difficult based only on DM if masses have round-like shapes and do not have architectural distortion, spiculated margins, or suspicious malignant or benign macrocalcification. The shape of these lesions demonstrated on DM images is similar, but the structure and density are very different, while these internal differences cannot be shown through DM (8). In traditional methods, the diagnosis of these diseases cannot rely on DM alone and must combine US or MRI. Previous studies (20-22,25) showed that radiomics provided massive features extracted from images to quantify tumors and allowed the possibilities for uncovering the differences that the human eye cannot recognize.

In this study, we aimed to investigate the most difficult

Table 5 DM characteristics for the differential diagnosis of TNBC and NTNBC

\begin{tabular}{lcccc}
\hline Characteristics variables & NTNBC & TNBC & $\chi^{2}$ value & P value \\
\hline Margin & 8 & 5 & & \\
Circumscribed & 23 & 18 & & \\
Obscured & & & 3.120 & 0.73 \\
Density & 1 & 0 & & 0.17 \\
Low-density & 16 & 7 & & \\
Equal-density & 14 & 16 & & \\
High-density & & & 5.480 & 0.065 \\
Location(depth) & 8 & 1 & & \\
Anterior & 16 & 12 & & \\
Middle & 7 & 10 & & \\
Posterior & & & & \\
\hline
\end{tabular}

DM, digital mammography; TNBC, triple-negative breast cancer; NTNBC, non-triple-negative breast cancer. and suspicious mass category. Thus, we focused on roundlike lesions with circumscribed or indistinct margins, without suspicious malignant or benign macrocalcifications. Our results showed that the DM-based prediction model for differentiating malignant and benign masses achieved high AUC values both in the training (0.94) and test groups $(0.88)$. It is worth mentioning that each feature in the model was significantly different between benign and malignant masses. Consistent with one previous study (23), combining DM with radiomics can significantly improve the diagnostic performance. According to the decision curve, we can see that patients could benefit from the entire risk threshold of 0 to 1 . The radiomics model in our study showed that the features derived from the MLO view were more abundant than those derived from the CC view, while the most highly weighted feature came from the $\mathrm{CC}$ view. One previous study (21) also showed that the features extracted from the MLO view achieved a higher prediction performance than those extracted from the CC view, indicating that the MLO view may provide more information than the $\mathrm{CC}$ view. The combination of the two views can provide more information than each of them individually (21). These features included 1 shape feature, 1 histogram feature, 1 texture feature, and 7 GLCM features. The proportion of GLCM features was the largest.

Furthermore, we demonstrated that the accuracy of the model for distinguishing round-like TNBC from NTNBC was 0.89 (training group) and 0.78 (in testing group), which is consistent with one previous study (21). While, there was no significant difference in DM characteristics between TNBC and NTNBC. The multivariable logistic regression model showed that the most predictive features included 1 histogram feature and 2 GLCM features. The proportion of GLCM features was larger as well, but the odds ratio value of the histogram feature was the largest. We only included the round-like mass, which is the most common presentation of TNBC. These results were encouraging because TNBC demonstrated rapid proliferation and poor

Table 6 Performance of the selected features discriminating TNBC and NTNBC

\begin{tabular}{lcccc}
\hline Selected feature & Coefficient & Odds ratio (95\% Cl) & P value & AUC value \\
\hline CC_kurtosis & 1.424 & $4.15[1.20-14.42]$ & $0.025^{\star}$ & 0.674 \\
CC_sumAverage & -1.551 & $0.21[0.06-0.80]$ & $0.022^{*}$ & 0.703 \\
MLO_InverseDifferenceMoment_AllDirection_offset4_SD & -1.185 & $0.31[0.12-0.81]$ & $0.017^{*}$ & 0.694 \\
\hline
\end{tabular}

TNBC, triple-negative breast cancer; NTNBC, non-triple-negative breast cancer; $\mathrm{Cl}$, confidence interval; AUC, area under the receiver operating characteristic curve; * means $\mathrm{P}<0.05$. 


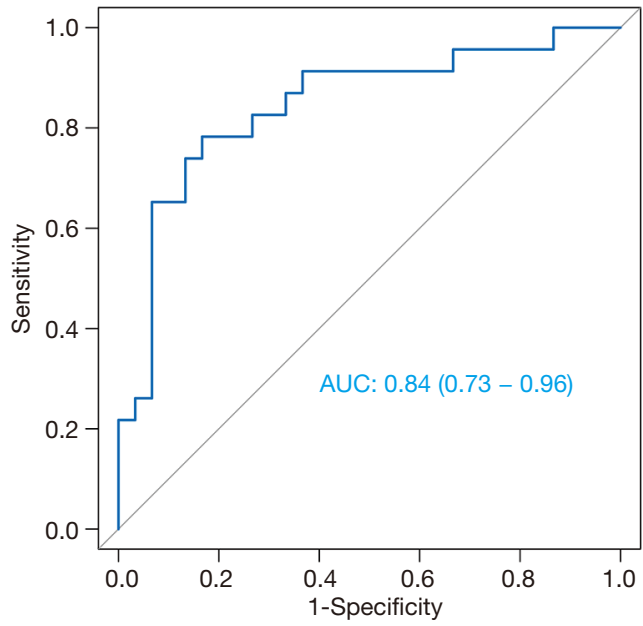

Figure 5 Receiver operating characteristic curves (ROC) of the model for predicting TNBC and NTNBC. TNBC, triple-negative breast cancer; NTNBC, non-triple-negative breast cancer.

Table 7 Cross-validation result of the model for predicting TNBC and NTNBC

\begin{tabular}{lccc}
\hline Group & Accuracy & Sensitivity & Specificity \\
\hline Training & 0.888 & 0.897 & 0.876 \\
Test & 0.783 & 0.794 & 0.767 \\
\hline
\end{tabular}

TNBC, triple-negative breast cancer; NTNBC, non-triplenegative breast cancer.

prognosis, and the risk of delayed or error diagnosis was higher.

In this study, the final constructed radiomics model included more GLCM features. The GLCM provides a second-order method for generating texture features to calculate the relationship between the combinations of gray levels in the image parameters (26), which can reflect internal spatial heterogeneity of the tumor lesions.

There are several limitations in this study. First, because there are no obvious boundaries between some diseased areas and normal tissues on DM images, no automatic segmentation algorithm for lesions is currently available. Thus, the lesion ROIs were manually segmented by 2 senior imaging physicians in consultation, but there may still be errors between the ROIs and the true boundary of the masses. Second, our study was an exploratory study, and the data were collected from a single institution, lacking validation from external cohorts. Third, we focused on only round-like masses, and the overall sample size of non-trinegative invasive ductal carcinoma was small. Therefore, no further molecular subtyping was performed for NTNBC. Last but not least, due to the small number of special pathological types of breast cancer in the study, it was impossible to present the ability of DM-based radiomics to distinguish among pathological types. Further research with more breast tumor cases and pathological subtypes is needed in the future.

\section{Conclusions}

In conclusion, our study demonstrates that without combining US and MRI examinations, DM-based radiomics can offer a more accurate discrimination between benign and malignant round-like masses. Additionally, DMbased radiomics for the prediction of TNBC or NTNBC in invasive ductal carcinoma achieved satisfactory accuracy. DM-based radiomics may have unique clinical value and provide quantitative information to aid radiologists in mammogram reading, clinical diagnosis and decisionmaking.

\section{Acknowledgments}

We thank American Journal Experts for their help in language editing. We thank Yaqiong $\mathrm{Ge}$ and $\mathrm{Pu}-\mathrm{Yeh} \mathrm{Wu}$ $(\mathrm{PhD})$ of GE Healthcare for their help in data statistics.

Funding: This work was supported by the Science and Technology Commission of Minhang District, Shanghai (grant number: 2019MHZ018).

\section{Footnote}

Reporting Checklist: The authors have completed the STARD reporting checklist. Available at http://dx. doi. org/10. 21037/gs-20-473

Data Sharing Statement: Available at http://dx. doi. org/10. 21037/gs-20-473

Peer Review File: Available at http://dx. doi. org/10.21037/ gs $-20-4733$

Conflicts of Interest: All authors have completed the ICMJE uniform disclosure form (available at http://dx. doi. org/10. 21037/gs-20-473). The authors have no conflicts of interest to declare. 
Ethical Statement: The authors are accountable for all aspects of the work in ensuring that questions related to the accuracy or integrity of any part of the work are appropriately investigated and resolved. The study was conducted in accordance with the Declaration of Helsinki (as revised in 2013). This retrospective study was approved by the institutional review board of Minhang Branch, Zhongshan Hospital (approval number: 2020-012-01K), and informed consent was waived.

Open Access Statement: This is an Open Access article distributed in accordance with the Creative Commons Attribution-NonCommercial-NoDerivs 4.0 International License (CC BY-NC-ND 4.0), which permits the noncommercial replication and distribution of the article with the strict proviso that no changes or edits are made and the original work is properly cited (including links to both the formal publication through the relevant DOI and the license). See: https://creativecommons.org/licenses/by-nc-nd/4.0/.

\section{References}

1. Coleman C. Early Detection and Screening for Breast Cancer. Semin Oncol Nurs 2017;33:141-55.

2. Gartlehner G, Thaler K, Chapman A, et al. Mammography in combination with breast ultrasonography versus mammography for breast cancer screening in women at average risk. Cochrane Database Syst Rev 2013;30:CD009632.

3. Waks AG, Winer EP. Breast Cancer Treatment. JAMA 2019;321:288.

4. Berry DA, Cronin KA, Plevritis SK, et al. Effect of screening and adjuvant therapy on mortality from breast cancer. N Engl J Med 2005;353:1784-92.

5. Oeffinger KC, Fontham ETH, Etzioni R, et al. Breast Cancer Screening for Women at Average Risk. JAMA 2015;314:1599.

6. Mercado CL. BI-RADS Update. Radiol Clin North Am 2014;52:481-7.

7. Thomassin-Naggara I, Tardivon A, Chopier J. Standardized diagnosis and reporting of breast cancer. Diagn Interv Imaging 2014;95:759-66.

8. Larribe M, Thomassin-Piana J, Jalaguier-Coudray A. Breast cancers with round lumps: Correlations between imaging and anatomopathology. Diagn Interv Imaging 2014;95:37-46.

9. Masood S. Breast cancer subtypes: morphologic and biologic characterization. Womens Health (Lond)
2016;12:103-19.

10. Foulkes WD, Smith IE, Reis-Filho JS. Triple-negative breast cancer. N Engl J Med 2010;363:1938-48.

11. Fulford LG, Reis-Filho JS, Ryder K, et al. Basal-like grade III invasive ductal carcinoma of the breast: patterns of metastasis and long-term survival. Breast Cancer Res 2007;9:R4.

12. Yang WT, Dryden M, Broglio K, et al. Mammographic features of triple receptor-negative primary breast cancers in young premenopausal women. Breast Cancer Res Treat 2018;111:405-10.

13. Wang Y, Ikeda DM, Narasimhan B, et al. Estrogen receptor-negative invasive breast cancer: imaging features of tumors with and without human epidermal growth factor receptor type 2 overexpression. Radiology 2008;246:367-75.

14. Dogan BE, Gonzalez-Angulo AM, Gilcrease M, et al. Multimodality Imaging of Triple Receptor-Negative Tumors With Mammography, Ultrasound, and MRI. AJR Am J Roentgenol 2010;194:1160-6.

15. Dogan BE, Turnbull LW. Imaging of triple-negative breast cancer. Ann Oncol 2012;23:vi23-9.

16. Turnbull LW. Dynamic contrast-enhanced MRI in the diagnosis and management of breast cancer. NMR Biomed 2009;22:28-39.

17. Hooley RJ, Scoutt LM, Philpotts LE. Breast ultrasonography: state of the art. Radiology 2013;268:642-59.

18. Bruening W, Uhl S, Fontanarosa J, et al. AHRQ Comparative Effectiveness Reviews. Noninvasive Diagnostic Tests for Breast Abnormalities: Update of a 2006 Review. Rockville (MD): Agency for Healthcare Research and Quality (US); 2012.

19. Parekh V, Jacobs MA. Radiomics: a new application from established techniques. Expert Rev Precis Med Drug Dev 2016;1:207-26.

20. Lei C, Wei W, Liu Z, et al. Mammography-based radiomic analysis for predicting benign BI-RADS category 4 calcifications. Eur J Radiol 2019;121:108711.

21. Ma W, Zhao Y, Ji Y, et al. Breast Cancer Molecular Subtype Prediction by Mammographic Radiomic Features. Acad Radiol 2019;26:196-201.

22. Zhou J, Tan HN, Bai Y, et al. Evaluating the HER-2 status of breast cancer using mammography radiomics features. Eur J Radiol 2019;121:108718.

23. Li Z, Yu L, Wang X, et al. Diagnostic Performance of Mammographic Texture Analysis in the Differential Diagnosis of Benign and Malignant Breast Tumors. Clin 
Breast Cancer 2018;18:e621-7.

24. Koo TK, Li MY. A Guideline of Selecting and Reporting Intraclass Correlation Coefficients for Reliability Research. J Chiropr Med 2016;15:155-63.

25. Gandomkar Z, Brennan PC, Mello-Thoms C, editors. A framework for distinguishing benign from malignant breast histopathological images using deep residual

Cite this article as: Wang L, Yang W, Xie X, Liu W, Wang H, Shen J, Ding Y, Zhang B, Song B. Application of digital mammography-based radiomics in the differentiation of benign and malignant round-like breast tumors and the prediction of molecular subtypes. Gland Surg 2020;9(6):2005-2016. doi: $10.21037 / g s-20-473$ networks. Fourteenth International Workshop on Breast Imaging; 2018.

26. Arebey M, Hannan MA, Begum RA, et al. Solid waste bin level detection using gray level co-occurrence matrix feature extraction approach. J Environ Manage 2012;104:9-18. 